Manuscript Number:

Title: Contact line curvature-induced molecular misorientation of a surface energy patterned organic semiconductor in meniscus-guided coating

Article Type: Full Length Article

Keywords: Molecular misorientation; surface energy patterning; organic semiconductor; meniscus-guided coating

Corresponding Author: Professor Jin-Hyuk Bae, Ph.D.

Corresponding Author's Institution: Kyungpook National University

First Author: Do-Kyung Kim

Order of Authors: Do-Kyung Kim; Premkumar Vincent; jaewon Jang; In Man Kang; Hyeok Kim; Philippe Lang; Muhan Choi; Jin-Hyuk Bae, Ph.D.

Abstract: The printing of organic semiconductors (OSCs) by means of meniscus guided coating (MGC) has great potential for the fabrication of high-performance, uniform, and large area flexible electronics. Furthermore, surface energy patterning allows low-cost, large area manufacturing, and is a requirement for device isolation to achieve accurate drive and to minimize power consumption. We investigated the morphological and electrical characteristics of surface energy patterned OSCs coated using the MGC method. A unique phenomenon, contact line curvature-induced molecular misorientation effect, which occurs in the patterning of OSCs with the MGC method, is demonstrated. This effect is highly dependent on the pattern width which decided contact line curvature. It influences the charge transport properties and hence the field-effect mobility of organic thin-film transistors (OTFTs). OTFTs in widths ranging from 50 to $500 \mu \mathrm{m}$ were fabricated. The highest misorientation angle was induced in the $50 \mu \mathrm{m}$ pattern width and the corresponding OTFTs exhibited the highest mobility. 


\section{Contact line curvature-induced molecular misorientation of a} 2 surface energy patterned organic semiconductor in meniscus3 guided coating

5 Do-Kyung Kim ${ }^{1}$, Premkumar Vincent ${ }^{1}$, Jaewon Jang ${ }^{1}$, In Man Kang ${ }^{1}$, Hyeok Kim², Philippe Lang ${ }^{3}$, Muhan $\mathrm{Choi}^{1}$, and

6 Jin-Hyuk Bae ${ }^{1}$

$8{ }^{1}$ School of Electronics Engineering, Kyungpook National University, Daegu, Korea

$9 \quad{ }^{2}$ Department of Electrical Engineering, Gyeongsang National University, Jinju, 52828, Korea

$10{ }^{3}$ ITODYS, Universite Paries Diderot CNRS UMR 7086 (Paris 7), Paris 75013, France

Full contact details of the corresponding author:

13 Prof. Jin-Hyuk Bae

14 Tel: (+82) 53-950-7222

15 Fax: (+82) 53-950-7222

16 Email address: jhbae@ee.knu.ac.kr 


\title{
17 Contact line curvature-induced molecular misorientation of a 18 surface energy patterned organic semiconductor in meniscus- guided coating
}

\begin{abstract}
The printing of organic semiconductors (OSCs) by means of meniscus guided coating (MGC) has great potential for the fabrication of high-performance, uniform, and large area flexible electronics. Furthermore, surface energy patterning allows low-cost, large area manufacturing, and is a requirement for device isolation to achieve accurate drive and to minimize power consumption. We investigated the morphological and electrical characteristics of surface energy patterned OSCs coated using the MGC method. A unique phenomenon, contact line curvature-induced molecular misorientation effect, which occurs in the patterning of OSCs with the MGC method, is demonstrated. This effect is highly dependent on the pattern width which decided contact line curvature. It influences the charge transport properties and hence the field-effect mobility of organic thin-film transistors (OTFTs). OTFTs in widths ranging from 50 to $500 \mu \mathrm{m}$ were fabricated. The highest misorientation angle was induced in the $50 \mu \mathrm{m}$ pattern width and the corresponding OTFTs exhibited the highest mobility.
\end{abstract}

\section{Keywords}

Molecular misorientation, surface energy patterning, organic semiconductor, meniscus-guided coating

\section{Introduction}

Great expectations for next-generation electronics have prompted active research on solution-processed organic thin-film transistors (OTFTs) which can be front-running candidates for low-cost, large-area device manufacturing, and flexible active-matrix displays and sensors ${ }^{1-3}$. High electrical performance beyond that of amorphous silicon TFTs has been achieved in solution-processed OTFTs through the tuning of small molecule organic semiconductors $(\mathrm{OSCs})^{4-7}$. One of the best tuning methods is to control the solution coating. In this regard, many solution coating techniques like inkjet printing, shearing, spincoating, spray coating, dip-coating, blade-coating, and slot-die coating have been developed ${ }^{8-14}$. Among these methods, meniscus guided coating (MGC) methods, as in blading, shearing, or slit-die coating, which deposit solution directionally on the target substrate by the virtue of capillary force between the substrate and the coating head, have been extensively studied because of their special advantages ${ }^{15}$. They can form uniform thin films with easily controlled film thickness in large-area fabrication compared to other coating methods. Moreover, they induce molecular alignments, and in some cases molecular packing due to their directionality. As a result, the charge transport properties of small molecule OSCs are enhanced and high field-effect mobility can be achieved".

Patterning of OSC thin films plays a significant role in the improvement of the electrical characteristics of OTFTs. Confinement of the OSCs through patterning suppresses leakage currents and crosstalk effect in integrated circuits, thus yielding more accurate TFT drive and lower power consumption ${ }^{16-18}$. These positive attributes have driven the development of various patterning methods for selective deposition of semiconductor thin films. Photolithography, template guided patterning, stamping, and chemical patterning have been introduced to pattern soluble OSCs ${ }^{19-21}$. In particular, chemical patterning that induces hydrophobic and hydrophilic region selectively through self-assembled monolayer formation or surface terminal group control has been widely studied due to its merits ${ }^{16-18,21}$. It is a facile method that does not require conventional photolithography and is not area constrained. This means that fewer process steps, lower manufacturing costs, and large-area fabrication can be achieved.

Nevertheless, many studies of MGC methods have been focused on improving unpatterned thin-film characteristics and TFT electrical performance by controlling coating velocity or solvents ${ }^{6,22,23}$. Although some reports have studied patterning methods and have fabricated patterned OTFTs, the morphological and electrical characteristics of MGC methods have not been fully understood. Furthermore, a unique 

methods should be addressed.

In this study, we investigated the morphological and electrical characteristics of the surface energy patterned bis (triisopropylsilylethynyl) (TIPS)-pentacene deposited with blade coating, one of the representative MGC methods. We observed the molecular misorientation that affected the charge transport properties between source and drain electrodes was induced in the mesoscale patterned layer by the contact line curvature of the blade coating process. In addition, the pattern width highly affected the contact line curvature-induced molecular misorientation. To demonstrate the electrical result of this effect, the OTFTs were fabricated with TIPS-pentacene thin films of various pattern widths. TIPS-pentacene thin films patterned with a $50 \mu \mathrm{m}$ width showed the lowest misorientation angle of $7.75^{\circ}$, while those patterned with a $300 \mu \mathrm{m}$ width exhibited the highest misorientation angle of $18^{\circ}$. Since the molecular misorientation affected their charge transport, OTFTs patterned with $300 \mu \mathrm{m}$ width showed the lowest field-effect mobility of $0.216 \mathrm{~cm}^{2} \mathrm{~V}^{-1} \mathrm{~s}^{-1}$. In contrast, the $50 \mu \mathrm{m}$ wide patterned OTFTs showed a relatively high field-effect mobility of $0.317 \mathrm{~cm}^{2} \mathrm{~V}^{-1} \mathrm{~s}^{-1}$, a more effective charge transport value.

\section{Materials and methods}

Figure 1(a) shows a schematic of the OSC patterning process. Indium-tin oxide (ITO)-deposited glass was prepared for the substrate and gate electrodes of the TFTs. Cross-linked poly(4-vinyl phenol) (c-PVP), mixed with methylated poly(melamine-co-formaldehyde) at a mass ratio of 1:1.25 and dissolved in propylene glycol methyl ether acetate at $10 \mathrm{wt}$. \% was spin coated on the ITO gates to form a polymer insulator. The substrate was spun at 3,000 revolutions per minute for $30 \mathrm{~s}$ and annealed at $200{ }^{\circ} \mathrm{C}$ for $1 \mathrm{~h}$ on a hot plate for cross-linking. For chemical patterning of the TIPS-pentacene, hydrophobic regions were generated through polydimethylsiloxane (PDMS) attachment on c-PVP and heated for 10 min at $120^{\circ} \mathrm{C}$. In this step, the methyl group, which is the surface terminal group of PDMS, was transferred onto the cPVP insulator ${ }^{18}$. To remove the methyl groups and generate the hydrophilic regions selectively, a shadow metal mask covered c-PVP surface was exposed to ultraviolet-ozone (UVO) for $10 \mathrm{~min}$. The detailed principles and the process of chemical patterning used in this study were described in our previous study ${ }^{18}$. Figure 1(b) describes the patterning process of TIPS-pentacene solution. The widths of the hydrophilic wetting regions were controlled in a mesoscale of $50-500 \mu \mathrm{m}$. Subsequently, a $2 \mathrm{wt}$. \% TIPS-pentacene solution dissolved in anisole was blade coated onto the c-PVP at $60{ }^{\circ} \mathrm{C}^{24}$. The TIPS-pentacene solution was captured between the blade and the substrate by capillary force and coated on the c-PVP insulator surface. It was coated directionally at various speeds of $0.1,0.3$, and $0.5 \mathrm{~mm} / \mathrm{s}$ and spontaneously deposited on the hydrophilic c-PVP region. An image of the patterned TIPS-pentacene is shown in Figure 1(c). Red arrows indicate the coating direction. Finally, $50 \mathrm{~nm}$ of Au was thermally deposited at a rate of $0.1 \mathrm{~nm} / \mathrm{s}$ to form the source and drain electrodes. Channel length was $100 \mu \mathrm{m}$ and the channel width depended on the patterned width of TIPS-pentacene thin films. The completed OTFTs were measured at room temperature under ambient pressure in a dark environment.

\section{Results and discussion}

To describe blade coated TIPS-pentacene structure and define the contact line curvature-induced molecular misorientation angle $\theta_{1}$, a 3D atomic force microscope (AFM) image of TIPS-pentacene was recorded as shown in Fig. 2. TIPS-pentacene was blade coated at the speed of $0.3 \mathrm{~mm} / \mathrm{s}$ with a substrate temperature of $60^{\circ} \mathrm{C}$, and a blade gap of $200 \mu \mathrm{m}$. For organic semiconductors, $\pi-\pi$ stacking structure of molecules facilitates charge transportt ${ }^{9,25}$. Theoretically, the $\pi-\pi$ stacking direction of solution-processed small molecules follows the direction of solution evaporation which is the same as the coating direction for MGC methods ${ }^{9,26}$. MGC methods form a well aligned and continuous molecular stacking of TIPSpentacene parallel to the coating direction due to their intrinsic directionality. Needle-shaped crystalline domains with $3 \mu \mathrm{m}$ width and $30 \mathrm{~nm}$ thickness were observed in this study as shown in Fig. 2. The long axis of the needle-shaped TIPS-pentacene crystalline domains was parallel to [210] as well as the crystal growth direction (blue arrow). Source and drain electrodes are usually designed to be parallel to the coating direction of OSC solutions for efficient field-effect charge transport., If molecular misorientation 
is induced, however, the unintended angular mismatch between the coating direction (red arrow) and crystal growth direction results in a disturbance of charge transport and a decrease in mobility. In Figure2, $\theta_{1}$ is the contact line curvature-induced misorientation angle which is the angle between coating direction and crystal growth direction.

The morphological characteristics of surface energy patterned TIPS-pentacene thin films were observed. Figure 3 display schematic and the corresponding cross-polarized optical microscope (CPOM) images of patterned TIPS-pentacene at varying pattern widths. Red arrows in the schematic and CPOM images indicate the coating direction, and the yellow arrow in the CPOM images indicates the crystal growth direction, which is the [210] direction of the TIPS-pentacene molecules. Regions 1 and 2 are the edge and middle sections of the patterned TIPS-pentacene thin films. The scale bars in the CPOM images are 50 $\mu \mathrm{m}$. The TIPS-pentacene solution was coated at different speeds of $0.1,0.3$, and $0.5 \mathrm{~mm} / \mathrm{s}$. As shown in Figure 3, the crystal texture and thickness vary with increasing coating speed, from long, wide and thick crystallites to short, narrow and thin crystallites. The molecular misorientation was observed in both regions 1 and 2 regardless of the coating speeds and widths.

To convey the effect of contact line curvature-induced misorientation on the pattern width, the contact line curvature-induced misorientation angles at various pattern widths are shown in Fig. 4(a). In this study, contact line curvature-induced misorientation angles and OTFTs were studied at a coating speed of 0.3 $\mathrm{mm} / \mathrm{s}$ because, at that speed, the highest crystallinity and best alignment of TIPS-pentacene were obtained, and as a result, the highest mobility of the OTFTs was observed ${ }^{27}$. The misorientation angle of unpatterned layers occurring due to fluid-flow was $9.87 \pm 2.13^{\circ}$. When the TIPS-pentacene was deposited on the confined hydrophilic wetting region of $500 \mu \mathrm{m}$ width, the misorientation angle increased to $15 \pm 3.61^{\circ}$. It increased more to $18 \pm 3.24^{\circ}$ at the pattern width of $300 \mu \mathrm{m}$. By contrast, it began to decrease with a decrease in the pattern width from $300 \mu \mathrm{m}$. The misorientation angles of $13.63 \pm 1.08^{\circ}$ and $7.75 \pm 1.14^{\circ}$ were observed at the widths of 100 and $50 \mu \mathrm{m}$, respectively. Interestingly, the misorientation angle of patterned TIPS-pentacene at $50 \mu \mathrm{m}$ width was smaller than that of the unpatterned layer. These results might have originated from the different contact line curvature of varying pattern widths.

Figure 4(b) shows several of the forces acting in a blade coating solution. First, capillary force helps in capturing the solution between substrate and blade head, and the surface tension makes a meniscus line. In addition, there is attraction between solution and wetting surface due to the hydroxyl group on the cPVP surface which generates high surface energy, so a solution in contact with the wetting surface remains on the wetting regions when the blade moves. There are dewetting regions outside of the wetting regions and the methyl group of the dewetting region forms a repulsive force between the hydrophobic surface and TIPS-pentacene solution (Figure 4(c)). The complexity of the forces and the different influences of these forces at varying pattern widths result in different contact line curvature-induced molecular misorientations while blade coating. When the pattern is narrow, as in the $50 \mu \mathrm{m}$ width, the solution on the wetting region is too small compared with the main solution captured between the blade and the surface, so the surface tension is dominant resulting in a high $\theta_{2}$ which is the angle induce the molecular misorientation in patterned layer. In addition, fewer nucleates in the wider patterns than in the unpatterned layer may induce well-oriented molecular stacking ${ }^{6}$. In summary, the patterned TIPS-

pentacene on the $50 \mu \mathrm{m}$ width showed the lowest misorientation angle $\theta_{1}$. When the pattern width is large, the amount of solution on the wetting region and the molecules sited at the center of the wetting region are predominantly affected by the attractive force but scarcely affected by the repulsive force. This results in a high contact line curvature, a small $\theta_{2}$, and a large $\theta_{1}$. As the pattern width increases beyond 300 , the repulsive forces cannot dominate most molecules except those sited at the edge due to the wide hydrophilic region, so the contact line curvature decreases again and results in a large $\theta_{2}$ and a small $\theta_{1}$ as shown in Figure 4(a).

We fabricated OTFTs to demonstrate the impact of contact line curvature-induced molecular misorientation on the OTFT electrical performance. Figure 5 (a)-(e) shows the transfer curves of the 50, 100,300 , and $500 \mu \mathrm{m}$ patterned OTFTs, and the unpatterned OTFTs coated at the speed of $0.3 \mathrm{~mm} / \mathrm{s}$. The corresponding field-effect mobilities were $0.317,0.240,0.216,0.267$ and $0.276 \mathrm{~cm}^{2} \mathrm{~V}^{-1} \mathrm{~s}^{-1}$. As the pattern width increased up to $300 \mu \mathrm{m}$, the mobility decreased from 0.317 to $0.216 \mathrm{~cm}^{2} \mathrm{~V}^{-1} \mathrm{~s}^{-1}$. In contrast, the mobility increased again from 0.216 to $0.276 \mathrm{~cm}^{2} \mathrm{~V}^{-1} \mathrm{~s}^{-1}$ as the pattern width increased from $300 \mu \mathrm{m}$. It exhibited a tendency opposite to the misorientation angle graph (Figure 4(a)) as shown in Figure 6. The 
contact line curvature-induced misorientation angle affected the charge transport properties and therefore the mobility of OTFTs.

\section{Conclusions}

We investigated the morphological characteristics and the molecular misorientation effect of surface energy patterned TIPS-pentacene deposited by means of blade coating. We demonstrated that contact line curvature-induced molecular misorientation occurs only in patterned OSCs coated using an MGC method and is highly dependent on the pattern width. The lowest molecular misorientation angle of $7.7^{\circ}$ occurred in a $50 \mu \mathrm{m}$ pattern width. This was due to the dominance of surface tension and the generation of fewer nuclei resulting in the highest mobility of $0.317 \mathrm{~cm}^{2} \mathrm{~V}^{-1} \mathrm{~s}^{-1}$. The highest misorientation angle was induced in a $300 \mu \mathrm{m}$ pattern width of due to the dominance of repulsive and attractive forces compared to other forces. The corresponding OTFTs exhibited the lowest mobility of $0.216 \mathrm{~cm}^{2} \mathrm{~V}^{-1} \mathrm{~s}^{-1}$. We expect this work will provide an optimized pattern width for small-molecule OSCs to achieve the most effective charge transport properties and to maximize the field-effect mobility of OTFTs.

\section{Acknowledgments}

This research was supported by the BK21 Plus project funded by the Ministry of Education, Korea (21A20131600011). This research was also supported by the Basic Science Research Program through the National Research Foundation of Korea (NRF) funded by the Ministry of Science and ICT (2018R1A2B6008815).

\section{References}

1. Kang, B., Lee, W. H. \& Cho, K. Recent advances in organic transistor printing process. ACS Appl. Mater. Interfaces 5, 2302-2315 (2013).

2. Yi, H. T., Payne, M. M., Anthony, J. E. \& Vitaly, P. Ultra-flexible solution-processed organic fieldeffect transistors. Nat. Commun. 3, 1259-1265 (2012).

3. $\mathrm{Xu}, \mathrm{W}$, et al. Flexible all-organic, all-solution processed thin film transistor array with ultrashort channel. Sci. Rep. 6, 29055-29061 (2016).

4. Diao, Y., et al. Solution coating of large-area organic semiconductor thin films with aligned singlecrystalline domains. Nat. Mater. 12, 665-671 (2013).

5. Kim, K., et al. A lattice-strained organic single-crystal nanowire array fabricated via solution-phase nanograting-assisted pattern transfer for use in high-mobility organic field-effect transistors. Adv. Mater. 28, 3209-3215 (2016).

6. Chang, J., Chi, C., Zhang, J. \& Wu, Jishan. Controlled growth of large-area high-performance smallmolecule organic single-crystalline transistors by slot-die coating using a mixed solvent system. Adv. Mater. 25, 6442-6447 (2013).

7. Li, H., et al. High-performance transistors and complementary inverters based on solution-grown aligned organic single-crystals. Adv. Mater. 24, 2588-2591 (2012).

8. Ha, J., et al. One-step interface engineering for all-inkjet-printed, all-organic componets in transparent, flexible transistors and inverters: polymer binding. ACS Appl. Mater. Interfaces 9, 8819-8829 (2017).

9. Giri, G., et al. Tuning charge transport in solution-sheared organic semiconductors using lattice strain. Nature 480, 504-508 (2011).

10. Yuan, Y., et al. Ultra-high mobility transparent organic thin film transistors grown by an off-centre spin-coating method. Nat. Commun. 5, 3005-3013 (2014). 
215 12. Jang, J., et al. Highly crystalline soluble acene crystal arrays for organic transistors: mechanism of 216 crystal growth during dip-coating. Adv. Funct. Mater. 22, 1005-1014 (2012).

217 13. Pierre, A., et al. All-printed flexible organic transistors enabled by surface tension-guided blade 218 coating. Adv. Mater. 26, 5722-5727 (2014).

219 14. Patel, B. B. \& Diao, Y. Multiscale assembly of solution-processed organic electronics: the critical 220 roles of confinement, fluid flow, and interfaces. Nanotechnology 29, 044004-044033 (2018). 15. Gu, X., Shaw, L., Gu, K., Toney, M. F. \& Bao, Z. The meniscus-guided deposition of semiconducting polymers. Nat. Commun. 9, 534-549 (2018).

16. Zhang, X., et al. Alignment and patterning of ordered small-molecule organic semiconductor micro/nanocrystals for device applications. Adv. Mater. 28, 2475-2503 (2016).

17. Li, Y., Sun, H., Shi, Y.\& Tsukagoshi, K. Patterning technology for solution-processed organic crystal field-effect transistors. Sci. Technol. Adv. Mater. 15, 024203-024027 (2014). 18. Kim, D. - K., et al. Importance of inherent and the relative surface energies in generating patterned layer in a solution process. J. Korean Phys. Soc. 68, 786-791 (2016).

19. Lee, E. K., et al. Chemically robust ambipolar organic transistor array directly patterned by photolithography. Adv. Mater. 29, 1605282-1605290 (2017).

20. Park, K. S., et al. Inkjet-assisted nanotransfer printing for large-scale integrated nanopatterns of various single-crystal organic materials. Adv. Mater. 28, 2874-2880 (2016).

21. Schmaltz, T., Sforazzini, G., Reichert, T. \& Frauenrath H. Self-assembled monolayers as patterning tool for organic electronic devices. Adv. Mater. 29, 1605286-1605310 (2017). resistance of 6,13-bis(triisopropylsilylethynyl) pentacene field-effect transistors fabricated by a modified flow-coating method. Appl. Phys. Lett. 100, 123301-123304 (2012).

23. Cour, I., et al. Origin of stress and enhanced carrier transport in solution-cast organic semiconductor films. J. Appl. Phys. 114, 093501-093508 (2013).

24. Bae, J. -H., et al. Thermal annealing effect on the crack development and the stability of 6,13bis(triisopropylsilylethynyl)-pentacene field-effect transistors with a solution-processed polymer insulator. Org. Electron. 11,784-788 (2010). 25. Wang. M, et al. High performance organic field-effect transistors based on single and large-area aligned crystalline microribbons of 6,13-dichloropentacene. Adv. Mater. 25, 2229-2233 (2013). 26. Janneck, R., Vercesi, F., Heremans, P., Genoe, J. \& Rolin, C. Predictive model for the meniscusguided coating of high-quality organic single-crystalline thin films. Adv. Mater. 28, 8007-8013 (2016). 27. Kim, D. -K., et al. Importance of angular mismatch on anisotropic field-effect mobility in solution- 
Figure 1. (a) Schematic illustration of the surface energy patterning process. (b) Schematic illustration of 281 the blade coating of TIPS-pentacene on pre-patterned C-PVP surface. (c) Photographic image of surface energy patterned TIPS-pentacene on c-PVP insulator. Red arrow indicates blade coating direction. 
(a)

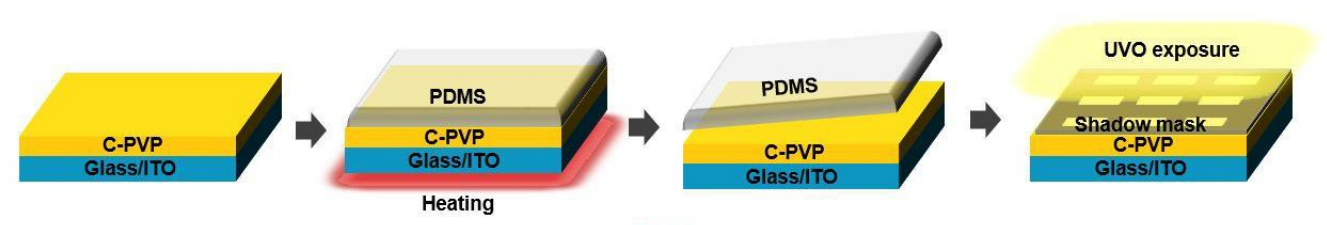

(b)

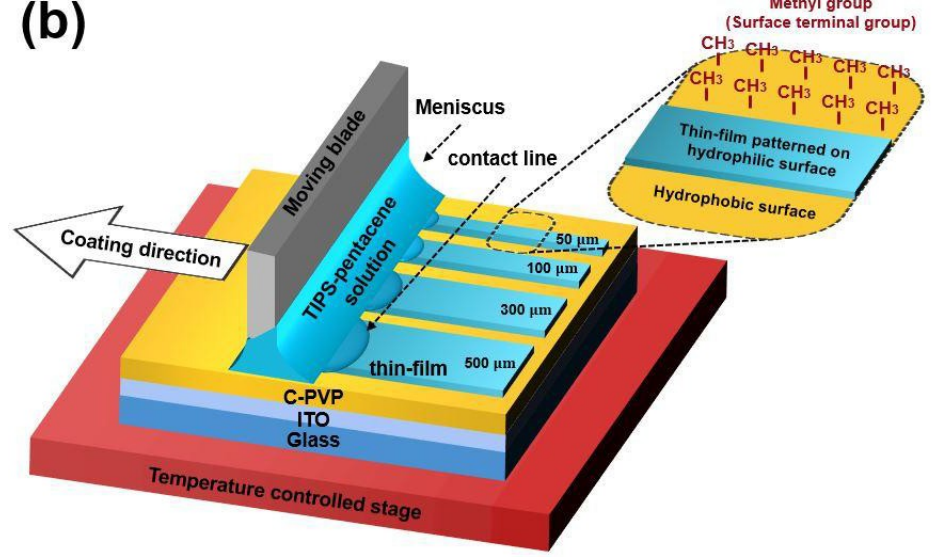

(c)

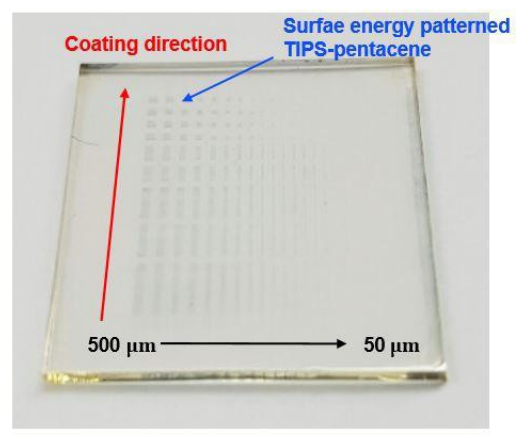


misorientation angle.

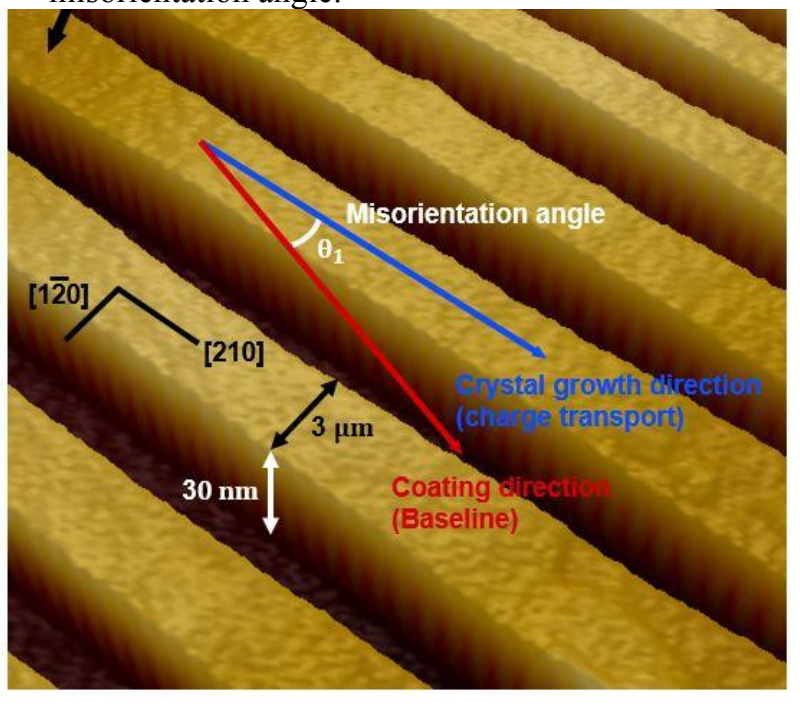

309

310

311

312

313

314

315

316

317

318

319

320

321

322

323

324

325

326

327

328

329

Figure 3. (a) Schematic illustration of patterned TIPS-pentacene thin films and (b) corresponding CPOM

330 images for various coating speeds and various widths. 
Region 1 (Edge)

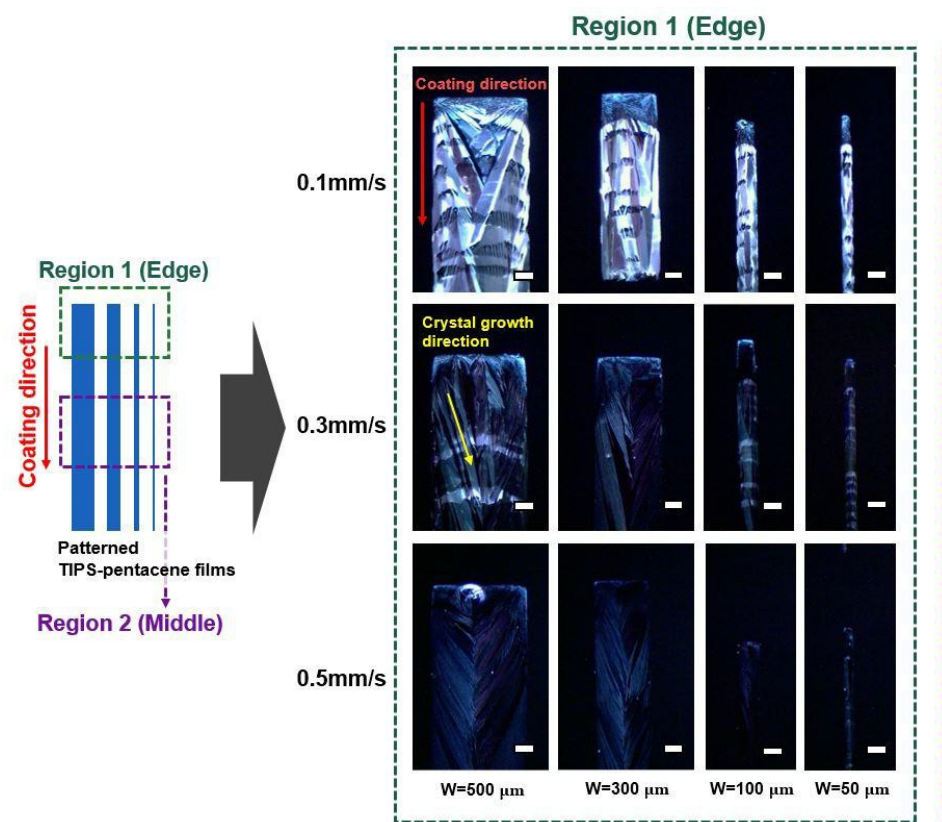

Region 2 (Middle)

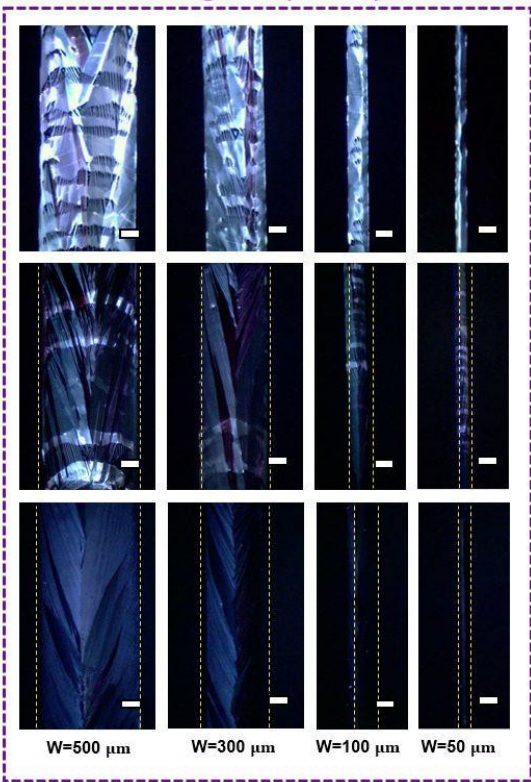

353 Figure 4. (a) Molecular misorientation angles of patterned TIPS-pentacene for various pattern width. 354 Schematic illustrations of (b) side view and (c) top view of meniscus-guided coating of TIPS-pentacene. 
(a)

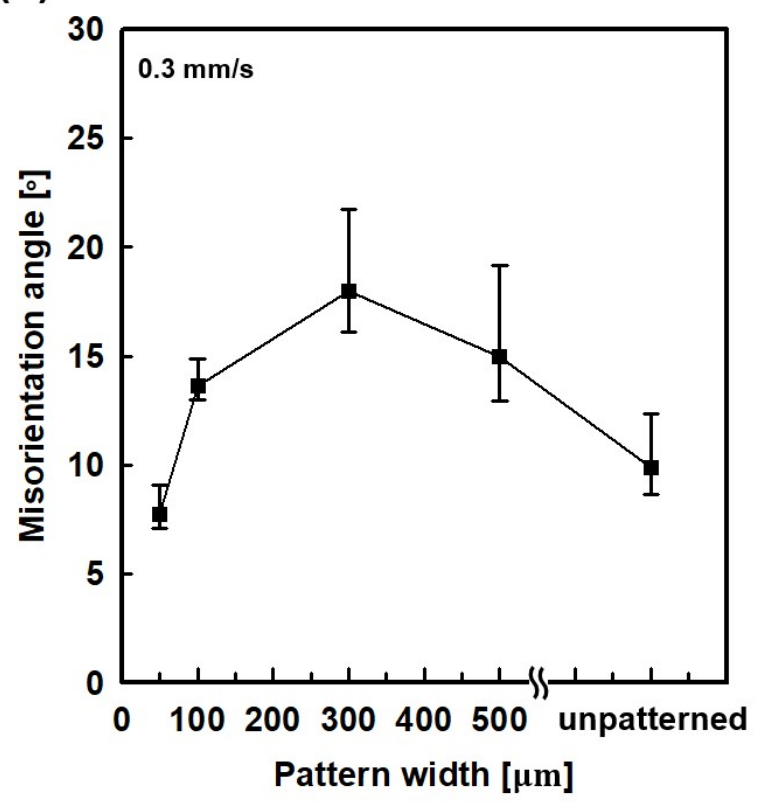

(b)

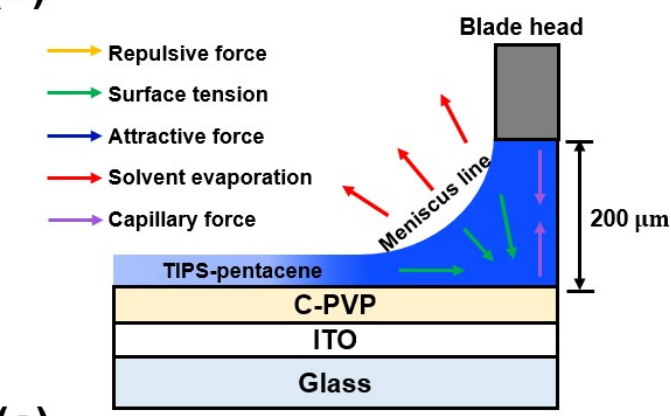

(c)

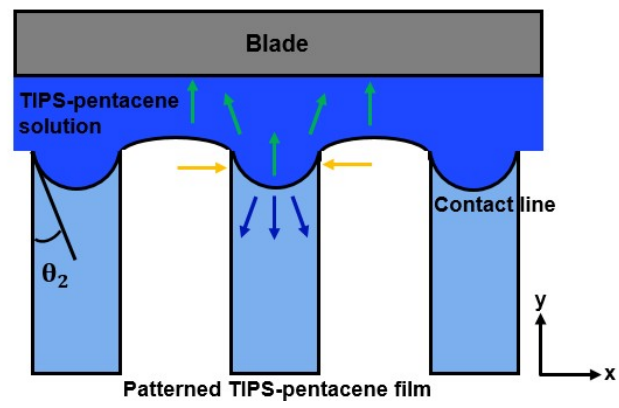


Figure 5. Representative transfer characteristic curves of the OTFTs coated at $0.3 \mathrm{~mm} / \mathrm{s}$ on the pattern

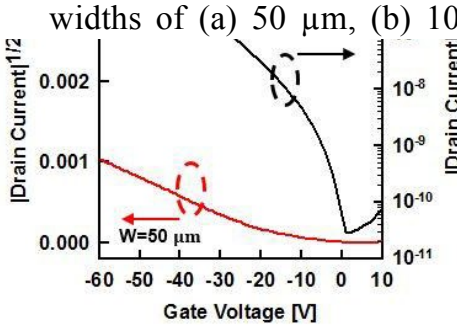

(d)

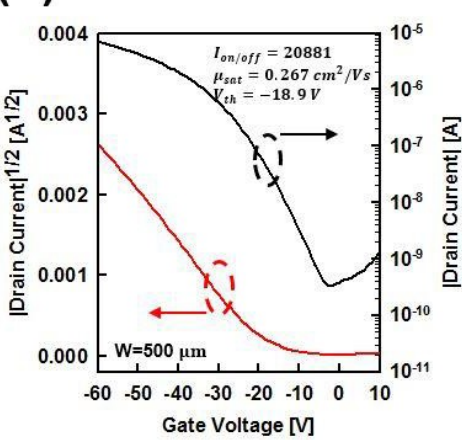

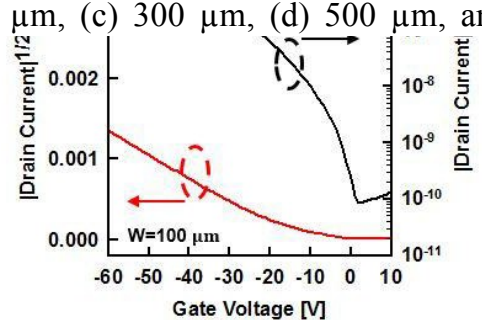

(e)
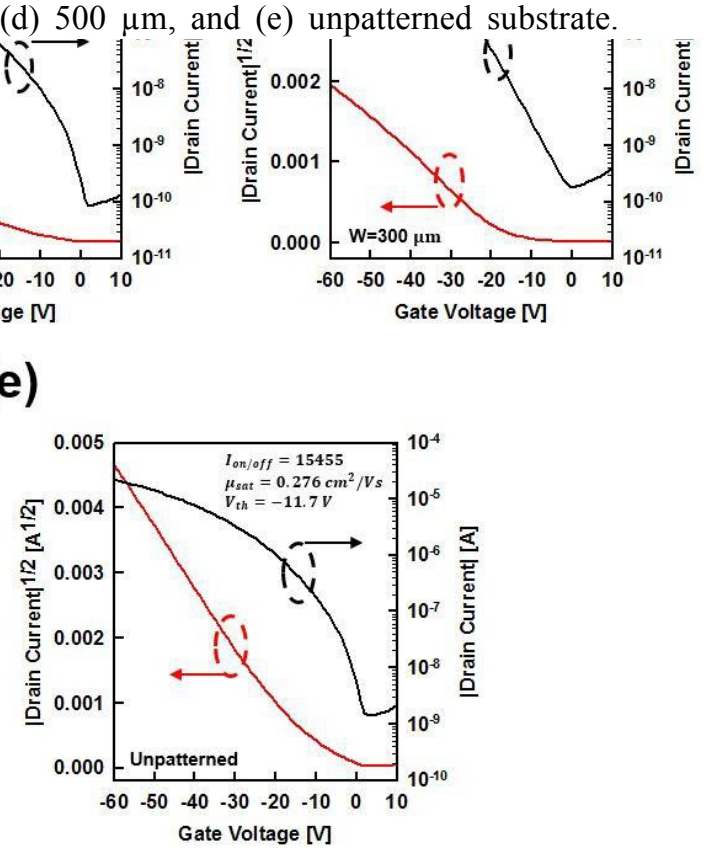

Figure 6. Average field-effect mobilities of OTFTs fabricated observed from surface energy patterned

TIPS-pentacene thin films for various pattern widths.

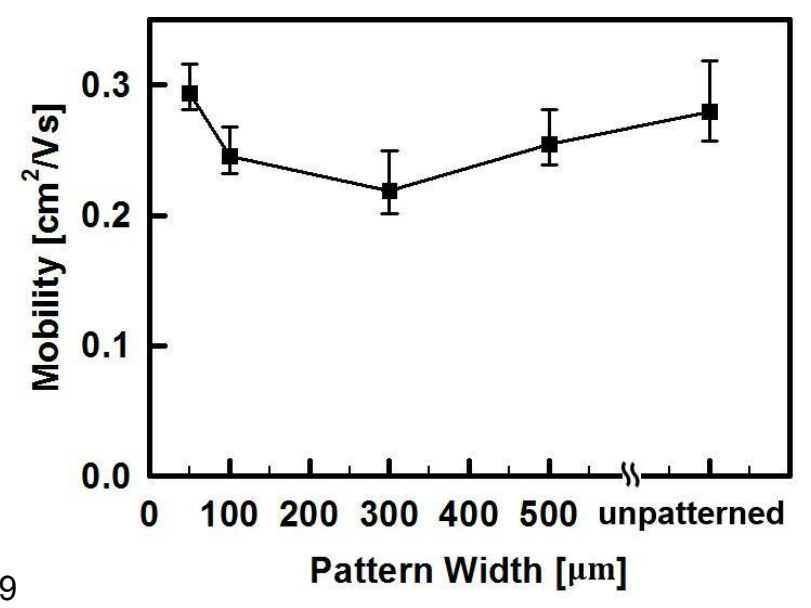

